\title{
Diacronie
}

Studi di Storia Contemporanea

$N^{\circ} 8,4 \mid 2011$

Le sembianze di Clio: approcci alla storia

\section{Lasciar parlare il silenzio. La Partition del subcontinente indiano e le abducted women}

\section{Piera Cavenaghi}

\section{OpenEdition}

\section{Journals}

\section{Edizione digitale}

URL: http://journals.openedition.org/diacronie/3530

DOI: $10.4000 /$ diacronie.3530

ISSN: 2038-0925

\section{Editore}

Association culturelle Diacronie

\section{Notizia bibliografica digitale}

Piera Cavenaghi, « Lasciar parlare il silenzio. La Partition del subcontinente indiano e le abducted women », Diacronie [Online], № 8, 4 | 2011, documento 4, Messo online il 29 octobre 2011, consultato il 30 avril 2019. URL : http://journals.openedition.org/diacronie/3530 ; DOI : 10.4000/diacronie.3530 


\title{
Diacronie
}

4/

\section{Lasciar parlare il silenzio. La Partition del subcontinente indiano e le abducted women}

\author{
Piera CAVENAGHI *
}

Le violenze perpetrate nel corso della Partition dell'India nel 1947 sono conosciute e documentate. Meno note sono le violenze che colpirono decine di migliaia di donne rapite e stuprate. Entrambi i governi di India e Pakistan ammisero questi crimini e si accordarono per ritrovare e reintegrare le donne rapite. Il governo indiano s'impegnò in modo particolare per portare a compimento il piano di recupero a costo di restituire le donne contro la loro volontà.

Il recupero delle donne rapite divenne una questione di onore nazionale che contrastò apparentemente con il concetto di onore familiare e della comunità. Molte delle donne riottenute furono infatti rifiutate dalle loro famiglie che le considerarono impure. Da parte sua il governo compì un grande sforzo per reintegrarle. Dopodiché il silenzio le avvolse e la memoria delle loro sofferenze svanì. I ricordi delle donne che si erano suicidate "per salvare il loro onore", invece, furono tramandati con enfasi.

\section{La Partition del Subcontinente indiano}

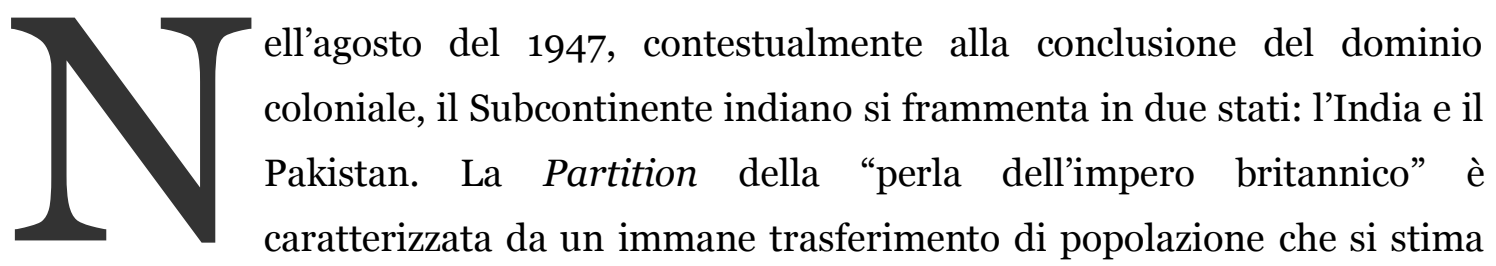
abbia coinvolto dodici milioni e mezzo di persone. Le province del Punjab e del Bengala sono letteralmente divise tra le due entità statali. In pochi mesi circa 5 milioni tra sikh e indù si spostano dal Punjab occidentale all'India e 5 milioni di musulmani affrontano il 
viaggio nel senso opposto ${ }^{1}$. Un movimento simile, anche se più modesto nella portata e nelle tragiche conseguenze, avviene tra il Bengala Occidentale e la sua parte orientale che diventa il Pakistan Orientale. La Partition è accompagnata da una violenza senza precedenti. Gli scontri intercomunitari che coinvolgono sikh e indù, da un lato, e musulmani, dall'altro, iniziano nell'agosto del 1946 per concludersi solo alcuni mesi dopo l'indipendenza. I massacri colpiscono soprattutto i villaggi a ridosso dei confini e i convogli di fuggitivi. In alcuni casi treni carichi di cadaveri giungono a destinazione provocando altre ondate di violenza. Si ritiene che le vittime siano da alcune centinaia di migliaia a un milione ${ }^{2}$.

Questo saggio riguarda le peculiari forme di abuso subite dalle donne nel corso della Partition e i significati attribuiti a tali violenze nel contesto della costruzione dei due nuovi Stati post-coloniali. Un aspetto rilevante è la connessione tra questi eventi e la nozione di onore familiare, comunitario e nazionale.

Nel 1989 vengono pubblicati i lavori di Urvashi Butalia, The Other Side of Silence e di Ritu Menon e Kamla Bhasin, Borders \& Boundaries3. Le autrici, attivamente impegnate nel movimento femminista indiano, mettono in piena luce, un aspetto della Partition, quello dei rapimenti e degli stupri avvenuti su entrambi i fronti, e in seguito gradualmente coperti dal silenzio. Come mettono in evidenza Ritu Menon e Kamla Bhasin, le violenze sulle donne non sono del tutto trascurate dalla storiografia tradizionale sulla Partition, né dai resoconti ufficiali, il problema, semmai, risiede nella prospettiva utilizzata, poiché le donne sono trattate come oggetti e non soggetti della storia ${ }^{4}$. Non tutti gli atti di violenza godono inoltre di pari dignità nell'essere ricordati: gli stupri, infatti, per le gravi ripercussioni sull'onore della comunità, subiscono un processo di espulsione dalla memoria ufficiale e di censura in quella privata. I suicidi per "salvare l'onore", al contrario, vengono tramandati in modo altisonante, ma anche in questo caso non si tratta altro che di un diverso modo di occultare e distorcere l'esperienza femminile della Partition.

È importante rilevare che oltre a rendere noti fatti fino a quel momento trascurati, le storiche si occupano di mettere in evidenza le implicazioni che essi hanno nella costruzione della nazione, uscendo così dall'ambito ristretto degli studi di genere e

\footnotetext{
${ }^{1}$ METCALF, Barbara, METCALF, Thomas, Storia dell'India moderna, Milano, Mondadori, 2004, p. 197.

2 Ibidem.

3 BUTALIA, Urvashi, The Other Side of Silence. Voices from the Partition of India, New Delhi, Penguin Books India, 1998; MENON, Ritu, BHASIN, Kamla, Borders \& Boundaries. Women in India's Partition, New Delhi, Kali for Women, 1998.

4 MENON, Ritu, BHASIN Kamla, Borders \& Boundaries. Women in India's Partition, New Delhi, Kali for Women, 1998, p. 11.
} 
dimostrando quanto tale prospettiva sia imprescindibile nella comprensione dei fenomeni storici. Accogliendo le riflessioni di Joan Jelly5, Menon e Bhasin, affermano che i medesimi avvenimenti possono essere vissuti diversamente da uomini e donne, e pertanto una storia narrata dalla sola angolazione maschile non può avere pretese di

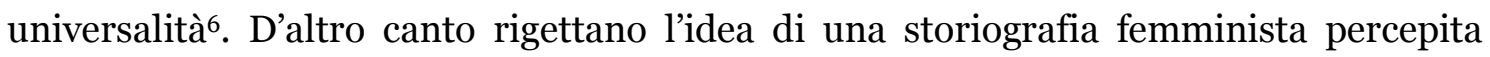
come un capitolo a parte ${ }^{7}$ e mirano ad un'integrazione delle prospettive di genere, la cui fusione porti ad interpretare il passato in modo complessivamente diverso. Un esempio di questo modo di operare viene offerto dalla studiosa pachistana Nighat Said Khan ${ }^{8}$, che muovendo dalla ricostruzione delle vite e dei rapporti intercomunitari delle donne musulmane precedenti la Partition, giunge a mettere in discussione l'assunto dell'inevitabilità religiosa e culturale di dividere la nazione indiana che è alla base dellideologia separatista musulmana e del movimento a favore della creazione del Pakistan.

La discussione teorica complessiva sulle periodizzazioni, le categorie di analisi sociale e le teorie dei mutamenti sociali è vivificata proprio dai problemi emersi dalla storia delle donne ${ }^{9}$ che pongono tra l'altro la questione fondamentale dell'uso e della validità delle fonti: le donne sono quasi assenti dai resoconti ufficiali di storia politica e economica perché questi non sono solitamente i loro ambiti d'azione. Le tracce dell'agire femminile vanno quindi cercate altrove: nelle testimonianze orali, o la memorialistica, per esempio. Va detto altresì che nel caso di Butalia, Menon e Bhasin l'operazione di raccolta dei dati e di scrittura viene svolta in modo particolarmente raffinato, combinando un'ampia gamma di fonti: interviste in profondità, rapporti e resoconti governativi, documenti privati, memorie, autobiografie, lettere, diari, registrazioni audio, dibattiti parlamentari e documenti legali ${ }^{10}$, evitando la contrapposizione tra documenti ufficiali e non, ma cercando invece di integrarli, offrendo così non solo informazioni, laddove è possibile, sulla dimensione del fenomeno da loro studiato, ma anche e soprattutto conoscenza sulla sua qualità e gli effetti sulle vite delle persone.

Il compito delle storiche della Partition è molto complesso perché la ricerca s' inoltra nel campo dell'indicibile, sia per il dolore provocato dalla rievocazione

5 KELLY, Joan, Women, History and Theory, Chicago, University of Chicago Press, 1984.

${ }^{6}$ MENON, Ritu, BHASIN, Kamla, Borders \& Boundaries. Women in India’s Partition, cit., p. 9.

7 Ibidem, p. 10.

8 SAID KHAN, Nighat, «Identity, Violence and Women: A Reflection on the Partition of India 1947", in VISWESWARAN, Kamala, Perspectives on Modern South Asia. A Reader in Culture, History, and Representation, Chichester, Wiley-Blackwell, 2011, pp. 134-138.

9 Joan Kelly citata in MENON, Ritu, BHASIN, Kamla, Borders \& Boundaries. Women in India's Partition, cit., p. 9.

${ }^{10}$ BHASIN, Kamla, Borders \& Boundaries. Women in India's Partition, cit., p. 17. 
dell'esperienza dell'abuso, sia per il muro di vergogna costruito intorno a questi avvenimenti che la società vuole in tutti i modi rimuovere. I silenzi, le omissioni nelle narrazioni familiari diventano allora segni che inducono a ricostruire le violenze del passato. A volte chi fa ricerca si confronta anche con la fiction narrativa $o$ cinematografica, utilizzandola quasi come “documento" valido non per la sua autenticità intrinseca, ma per aver colto e mantenuto in vita tracce di vissuti altrimenti destinati a scomparire dalla memoria ${ }^{11}$. Va sottolineato infatti che di fronte alla lacuna durata un quarantennio della ricerca sulle violazioni delle donne, la narrativa nella forma di novelle, romanzi e, in seguito film, non ha mai smesso di "ricordare" svolgendo così un insostituibile ruolo di supplenza.

\section{Le abducted women}

Durante la Partition decine di migliaia di donne vengono rapite e stuprate, costrette a partorire figli che sono poi loro sottratti, mutilate, forzatamente convertite, forzatamente sposate, marchiate con i simboli religiosi dei loro aguzzini, ma anche assassinate, o indotte al suicidio dai loro stessi familiari.

Tali violenze, caratterizzate da estrema brutalità sessuale, sono anche discorsi scritti sui corpi delle donne attraverso i quali parlare al nemico col quale, nonostante la volontà di segnare confini e differenze si condividono lingua, costumi e codici etici.

Queste violenze, infatti, - rimarcano Menon e Bhasin - sono scioccanti anche per ciò che ci dicono sulle costruzioni ideologiche maschili intorno all'onore ${ }^{12}$.

Inserite nell'ambito della comune cultura patriarcale, le norme legate all'onore maschile, di cui la sessualità femminile non è altro che un'espressione della mascolinità, dettano legge anche in frangenti di estremo disordine e nel successivo recupero della quotidianità da parte di famiglia, comunità e Stato.

I testimoni e gli autori di violenze innominabili spiegheranno tanta brutalità come una sorta di patologia di quel momento storico ${ }^{13} \mathrm{ma}$, pur nella follia, quegli atti di

\footnotetext{
${ }^{11}$ Sul dibattito sul ruolo della fiction nella narrazione delle violenze di genere nella Partiton si veda: DIDUR, Jill, «At a Loss for Words: Reading the Silence in South Asian Women's Partition Narratives», in Topia, 2000, 4 (Fall), 53-71, URL: < http://pi.library.yorku.ca/ojs/index.php/topia/article/view/160/150 >, [consultato il 20 agosto 2011];

DIDUR, Jill, Fragments of Imagination: Re-thinking the Literary in Historiography through Narratives of India's Partition [on-line],

URL: < http://english.chass.ncsu.edu/jouvert/vii2/DIDUR.HTM >, [consultato il 12 agosto 2011].

12 Ibidem, p. 43.
} 
violenza seguono in modo esasperato una sorta di grammatica che potremmo chiamare "sistema dell'onore", valida in tempi di pace quanto in quelli di guerra. Più che di rottura, Menon e Bhasin affermano che si tratta di un continuum di violenze messe in connessione dal consenso generale sul tema delle violenza esercitata sulle donne, consenso che collega la violenza sessualizzata contro le donne del nemico con quella esercitata dai loro stessi parenti per salvarle e infine quella perpetrata dallo Stato nel suo "apparentemente benefico" programma di recupero ${ }^{14}$.

\section{Le operazioni di recupero nella cornice dell'onore: le abducted women tra azione dello Stato e accettazione della comunità}

I rapimenti e stupri di donne su entrambi i fronti iniziano già nel 1946, l'anno precedente l’indipendenza (14 agosto per il Pakistan e 15 agosto del 1947 per l'India). L'Indian National Congress ${ }^{15}$, ne dà notizia nella sessione del 23-25 novembre 1946 a Meerut e chiede che vengano prese delle misure per ritrovare le donne rapite e riportarle nelle loro case. Nella medesima risoluzione si afferma che le conversioni di massa non hanno significato o validità e che "people must be given every opportunity to return to the life of their choice"16.

I sequestri subiscono un'impennata dal marzo dell'anno successivo e ciò desta un tale allarme che in settembre le delegazioni dei governi di Pakistan e India s'incontrano per accordarsi sulla reciproca restituzione delle donne rapite e rilasciano una dichiarazione congiunta circa l'invalidità delle conversioni e dei matrimoni forzati seguiti ai sequestri ${ }^{17}$. Questo sforzo unitario prosegue il 6 dicembre del 1947 quando le due potenze convocano una Interdominion Conference per rendere più efficaci le azioni di recupero. Segue un ulteriore meeting l'11 novembre del 1948 che fissa i termini delle operazioni e sia l'India che il Pakistan emettono delle specifiche ordinanze.

\footnotetext{
13 «We simply went mad» risponde Harjit, un sikh che vive presso la città di Atari, cercando di spiegare la ragione di una tale violenza scoppiata tra gente che fino a quel momento aveva vissuto, spesso in buoni rapporti, l'una accanto all'altra. BUTALIA, Urvashi, The Other Side of Silence. Voices from the Partition of India, cit., p. 73.

14 MENON, Ritu, BHASIN, Kamla, Borders \& Boundaries. Women in India's Partition, cit., p. 58.

${ }_{15}$ La principale organizzazione indiana che aveva guidato la lotta contro il colonialismo britannico, divenuta dopo l'indipendenza il partito di maggioranza fino al 1977.

16 MENON, Ritu, BHASIN, Kamla, «Abducted Women and the Questions of Honour», in JAYAWARDENA, Kumari, DE ALWIS, Malathi, Embodied Violence: Communalising Women's Sexuality in South Asia, New Delhi, Kali for Women, 1996, p. 6.

${ }_{17}$ BUTALIA, Urvashi, The Other Side of Silence. Voices from the Partition of India, cit., p. 13
} 
Le stime ufficiali sostengono che siano state 50.000 le donne musulmane sequestrate in India e 33.000 quelle indù e sikh in Pakistan ${ }^{18}$. In base ad altri calcoli, si ritiene che le donne rapite siano state complessivamente $100.000^{19}$.

Le abducted women subiscono destini molto diversi: alcune sono violentate, costrette a portare avanti la gravidanza per vedersi poi sottrarre i figli appena nati ${ }^{20}$, altre sono forzatamente convertite e sposate dai loro rapitori, altre ancora vendute e/o avviate alla prostituzione.

Malgrado India e Pakistan siano nazioni belligeranti a causa dei territori del Kashmir e vi siano numerosissime altre ragioni di contesa, i due governi si trovano d'accordo nell'ammettere le reciproche responsabilità riguardo ai rapimenti e nell'organizzare un piano che permetta di accogliere nei rispettivi territori di ufficiali dell'altro paese preposti alla ricerca delle donne scomparse.

La divisione dell'India si realizza sulla base di un nazionalismo religioso e culturale che fa sì che, in contrasto con la realtà sociologica del subcontinente indiano, il Pakistan divenga "la nazione dei musulmani indiani" e, di conseguenza, l'India "la nazione degli indù”. Su questo assunto gli Stati concordano sul fatto che i matrimoni avvenuti tra una donna di una religione e un uomo dell'altra siano nulli e che la donna debba essere riportata a casa propria, ossia in quello che è divenuto il luogo delle propria appartenenza religiosa ${ }^{21}$.

Il governo indiano, in particolare, si applica alle operazioni di recupero con singolare energia e dispendio di mezzi ${ }^{22}$. Nell'azione di ricerca sono coinvolti, infatti, numerosi corpi dello Stato come: l'Ufficio del Deputy High Commissioner, il Military Evacuation Office, il Chief Liaison Officer, e la Organization for Recovering Abducted Women, organismo costituito da volontarie ed altri ufficiali, appositamente creato per adempiere al compito di ritrovare e rimpatriare le donne rapite. La responsabilità dell'organizzazione è affidata a Mridula Sarabhai ${ }^{23}$ che come altre operatrici proviene

\footnotetext{
${ }^{18}$ Ibidem, p. 7.

19 KHOSLA, Gopal Das, citato in DAS, Veena, Critical Events. An Anthropological Perspective on Contemporary India, Oxford, Oxford University Press, 1995, p. 59.

${ }^{20}$ BUTALIA, Urvashi, The Other Side of Silence. Voices from the Partition of India, cit., p. 132.

${ }^{21}$ Ibidem, p. 139.

${ }_{22}$ BASU, Aparna, Mridula Sarabhai: Rebel with a Cause, Oxford, Oxford University Press, 1996, p. 125 .

${ }^{23}$ Mridula Sarabhai (1911-1974), è fin da giovanissima una seguace di Gandhi e attivista del Congress Party presso il quale ricopre incarichi di rilievo. Nel 1947, in occasione dell'InterDominion Conference, pur in assenza di una nomina ufficiale, viene deputata a organizzare le attività di recupero delle donne rapite. Mossa da una devozione e imparzialità assolute, la causa acquista per lei il carattere di azione politica che la induce a forzare i rimpatri delle donne anche a dispetto della loro volontà. Ha un ruolo di primaria importanza nella definizione delle Abducted Persons (Recovery and Restoration) Act del 1949. Per approfondimenti sulla figura di Mridula Sarabhai si veda: BASU, Aparna, op. cit.
} 
da un retroterra d'impegno nel movimento gandhiano. Agli organi di polizia si accordano ampi poteri per rintracciare e rimpatriare le donne. Un tribunale congiunto viene preposto alla discussione dei casi dubbi.

Gli sforzi non si limitano alla ricerca e al rimpatrio delle donne, ma vengono organizzati anche dei centri di raccolta per le donne ritrovate (ashram) e, nel tempo, corsi di qualificazione professionale.

La maggior parte dei recuperi è effettuata dal settembre del 1947 al luglio del 1948, dopo questa data subisce una diminuzione in seguito al ritiro del Military Evacuation Office. Le ricerche e i ritrovamenti proseguono tuttavia fino al 1956.

Perché un tale coinvolgimento da parte dello Stato? L’Abducted Women Recovery Operation è investita di molteplici significati e diviene una questione di onore nazionale. Nella prospettiva indiana la cessione da parte dell'Indian National Congress di una parte del territorio al Pakistan è percepito come la mutilazione del corpo dell'antica India. Ciò viene accettato come l'ineluttabile prezzo per ottenere l'indipendenza, non è però accettato il ratto delle donne, il cui recupero è inteso come la compensazione della perdita territoriale, il recupero di una proprietà ${ }^{24}$. Le autorità sono tempestate furono oggetto dalle istanze dei familiari che chiedono loro di ritrovare le donne scomparse e lo Stato si ritrova quindi investito del ruolo di parens patriae.

Grazie alla mobilitazione dello Stato molte donne vengono felicemente ricongiunte alle loro famiglie, ma la realtà di questi ritrovamenti presenta anche delle gravi criticità che affiorano ancora una volta dalla narrativa e dalle memorie personali delle vittime e delle volontarie che si sono impegnate nell'operazione di recupero. Queste informazioni non sarebbero mai venute alla luce attraverso la lettura delle sole statistiche o dei rapporti ufficiali ed è arduo quantificarle, ciò nondimeno rendono conto delle problematiche che tale operazione incontra.

Innanzitutto, non tutte le donne ritrovate vengono accettate dalle famiglie d'origine per una sorta di presunzione di colpa per cui le vittime sono ritenute irreparabilmente contaminate dal fatto di essere state in contatto - e probabilmente stuprate - dagli Altri, i musulmani. Questi ripudi devono assumere delle proporzioni consistenti tanto è vero che sia il primo ministro Jawarharlal Nehru, sia il Mahatma Gandhi sentono il dovere di lanciare degli appelli, in cui affermavano che le donne recuperate sono ancora pure e devono essere trattate come sorelle ${ }^{25}$.

24 MOOKERJEA-LEONARD, Debali, «Quarantined: Women and the Partition», in Comparative Studies of South Asia, Africa and the Middle East, 24, 1/2004, p. 33.

25 BUTALIA, Urvashi «Abducted and Widowed Women: Questions of Sexuality and Citizenship During Partition», in MEENAKSHI Thapan (ed.), Embodiment: Essays on Gender and Identity: 
Gandhi si pronuncia sulla questione dei rapimenti delle donne fin dall'autunno del 1946, quando i disordini intercomunitari scoppiano in un India ancora indivisa. Fin da questo momento è solidale con le ragazze rapite e rifiutate dalla famiglia dichiarando che non deve essere impedito loro il ritorno a casa, né sono necessari riti di purificazione, o penitenze e che la società indù è ingiusta con queste persone poiché "They had not erred." 26 . Gandhi ritorna più volte sull'argomento sollecitando le famiglie ad accogliere le donne e si spinge fino al punto di affermare senza mezzi termini che sono i violentatori che si dovrebbero vergognare, e non le "povere ragazze" 27. Dichiarazione molto forte, questa, in un contesto culturale in cui la donna è considerata l'unica responsabile della difesa della castità di fronte ad ogni evenienza. A fronte, tuttavia, di una difesa inequivocabile delle donne rapite il pensiero di Gandhi diventa, come vedremo, apparentemente contraddittorio riguardo il prezzo da pagare per la difesa della purezza femminile.

Il riacquisto delle donne è inserito in un sistema di riferimenti mitologici tra cui spicca l'epopea di Rama alla ricerca della consorte, Sita. Il richiamo al Ramayana, uno dei più importanti poemi della letteratura classica indiana, in tanti pamphlet fatti circolare per l'occasione, si riferisce al fatto che il governo si sente chiamato a cercare le proprie donne come a un dovere sacro, e a sostenere l'argomento della riammissione di queste presso le famiglie poiché esse, come Sita, vanno considerate incontaminate ${ }^{28}$. Il Ramayana però non contiene solo questi significati e può essere utile esplorarne brevemente il contenuto: la regina Sita, moglie di Rama viene rapita dal demone Ravana e nascosta nell'isola di Lanka. Rama dopo varie vicende sconfigge Ravana e ritrova Sita, ma deve ripudiarla perché la regina è stata costretta a sedersi sulle ginocchia di Ravana e ciò ne mette in dubbio la purezza. Sita si sottopone allora alla prova del fuoco e, superatala, è riaccolta dal marito.

In epoca medievale, però, si aggiunge un secondo finale in cui Sita viene nuovamente ripudiata da Rama. Egli cede alle pressioni dei suoi concittadini. Seguendo il suo esempio, infatti, tutti avrebbero dovrebbero riaccogliere donne sospette di vari gradi d’impurità compreso l'adulterio ${ }^{29}$. Molti anni dopo Rama sembra disposto a riprendere la sposa in cambio di un giuramento sulla sua purezza. Come atto estremo di

URL: < http://jan.ucc.nau.edu/ sj6/butaliaabductedwomen.pdf >, [consultato 16 agosto 2011]. ${ }^{26}$ GANDHI, Mohandas K., "Speech at Prayer Meeting», in The Collected Works of Mahatma Gandhi [Electronic Book], vol. 92, New Delhi, Publications Division Government of India, 1999, p. 367.

27 «It is the men who commit rape that should feel ashamed, not these poor women». GANDHI, Mohandas K., «Speech at Prayer Meeting», in cit., vol. 97, p. 399.

${ }^{28}$ BUTALIA, Urvashi, The Other Side of Silence. Voices from the Partition of India, cit., p. 160.

29 Ringrazio Elisa Freschi, research fellow presso il dipartimento di Studi Orientali dell'Università 'La Sapienza' di Roma, per i chiarimenti circa le evoluzioni del Ramayana. 
devozione e innocenza Sita chiede di essere accolta dalla Madre Terra ed è accontentata $^{30}$. Sita non si toglie la vita, tuttavia va rimarcata l'ossessiva richiesta rivolta alla regina di dar prova della propria castità di fronte ad un abuso solo sospettato e di cui in ogni caso è stata la vittima. Ciò che è in gioco è l'apparato dell'onore su cui ruota la struttura sociale indiana: in base a tale sistema il fulcro dell'onore patriarcale maschile risiede nel controllo e nella protezione della purezza delle sue donne. Il solo dubbio sulla castità femminile diviene un elemento di realtà. Una volta che la purezza di una donna viene anche solo messa in discussione è irreparabilmente compromessa e, se da un lato il Ramayana è la celebrazione di Sita come il modello esemplare della castità femminile, dall'altro, soprattutto nella versione medievale, ci racconta che non c'è possibilità per Sita e Rama di ritornare alla vita di prima e curare il dolore. Per Sita l'unico modo per farsi rispettare è quello di essere riassorbita dalla Terra e non rimanere nel mondo degli umani. Sebbene sappia che Sita è rimasta pura e che Rama desidera riunirsi a lei, la società avanza i propri diritti sulla vita della coppia. L'onore e il prestigio sono un fatto sociale, non privato e non vi è nessuna considerazione delle sofferenze delle vittime. Lo Stato-Rama nulla può contro l'opinione dei cittadini la cui mascolinità e il cui onore dipendono dalla purità delle proprie donne, ma al tempo stesso fa parte dei doveri e dell'onore dello Stato, come di Rama, recuperare le proprie donne anche se poi ne verrà messa in dubbio l'onestà. Seguendo il parallelo Stato-Rama si potrà comprendere l'azione dello Stato indiano e dei suoi cittadini nella conduzione della Recovery Operation.

Che esista la concreta possibilità di essere rifiutate dalle famiglie è un fatto paventato dalle stesse vittime che, una volta ritrovate e condotte nei campi di accoglienza manifestano la loro ansietà alle assistenti sociali. Queste ultime sono solite rassicurale dicendo loro che sono figlie dell'India, figlie del Pandit Nehru e che le istituzioni si prenderanno cura di loro ${ }^{31}$. Altre donne si dimostrano riluttanti nel voler ritornare a casa, chiedendo di rimanere coi loro rapitori. Per ovviare a queste resistenze, all’inizio del 1948, Pakistan e India si accordano affinché in tali casi le donne siano "evacuate a forza", laddove per tutti gli altri rifugiati, si è stabilito di non forzare il rientro fino a quando la sicurezza non sia stata ripristinata del tutto32.

Il governo indiano apre a Jalandhar, Amristar, Karnal e Delhi dei centri di accoglienza specifici per ospitare le donne rimpatriate (ashram) e in attesa di essere

30 PISANI, Vittore, LAXMAN PRASAD Misra, Le letterature dell'India, Milano, BUR, 1993, pp. 81-85. ${ }^{31}$ MENON, Ritu, BHASIN, Kamla, «Abducted Women and the Questions of Honour», cit., p. 19. ${ }^{2}$ BUTALIA, Urvashi, The Other Side of Silence. Voices from the Partition of India, cit., pp. 157158. 
accolte dalle proprie famiglie. Per parte di queste donne, rifiutate dalle famiglie che non vogliono accogliere donne contaminate, magari per il solo fatto di aver vissuto sotto la protezione di una famiglia musulmana33, questi ashram divengono vere e proprie case per lungo tempo, se non per sempre 34 .

In altri frangenti le donne possono essere reintegrate a patto che riescano a trasmettere l'idea di essere rimaste incorrotte, ma la cosa si complica moltissimo in presenza di figli sbagliati, nati dall'unione con il nemico. Dal punto di vista simbolico il corpo-confine delle donne, contaminato dal nemico, diventa anche un corpo traditore perché porta entro i confini dell'India i figli dell'Altro35.

Kamblabehn Patel, braccio destro di Mridula Sarabhai, descrive l'intervento dello Stato nello svolgere il compito di "ripulire" le donne ingravidate dal nemico ${ }^{36}$. Esse vengono ospitate nel campo di Jalandhar per un periodo di tre mesi durante il quale

33 MOOKERJEA-LEONARD, Debali, op. cit., p. 39.

34 BUTALIA, Urvashi, The Other Side of Silence. Voices from the Partition of India, cit., pp. 162163.

35 MENON, Ritu, BHASIN, Kamla, Borders \& Boundaries. Women in India's Partition, cit., p. 44. La presenza di figli nati dal nemico è un elemento di gravissima contraddizione in tutte le realtà in cui si sono verificati degli stupri di guerra. Riflettendo sul conflitto nella ex-Yugoslavia alcune giuriste femministe parificheranno gli stupri e le conseguenti gravidanze forzate al reato di genocidio. MACKINNON, Catherine A., "Rape, Genocide, and Women's Human Rights», in STIGLMAYER, Alexandra (ed.), Mass Rape: the War against Women in Bosnia-Herzegovina, Lincoln, University of Nebraska Press, 1994, pp. 183-196.). Il paradosso di tale approccio è, come osserva Bruna Bianchi, che finisce per riflette gli stereotipi patriarcali ed etnicisti propri di chi ha perpetrato le violenze. I figli, infatti, sono considerati solo del padre di cui trasmetterebbero i caratteri e la contaminazione etnica. BIANCHI, Bruna, «Introduzione», in DEP. Rivista telematica di studi sulla memoria femminile: Genere, nazione, militarismo. Gli stupri di massa nella storia del Novecento e nella riflessione femminista [pfd], Venezia, 10/2009, pp. I-XVI, URL: < http://www.unive.it/media/allegato/dep/n10-2009/Dep 010.pdf $>$, [consultato il 30 agosto 2011];

DEP. Rivista telematica di studi sulla memoria femminile: Genere, nazione, militarismo. Gli stupri di massa nella storia del Novecento e nella riflessione femminista [pdf], Venezia, 10/ 2009, URL: < http://www.unive.it/media/allegato/dep/n10-2009/Dep_010.pdf >, [consultato il 30 agosto 2011].

36 Ibidem. Le autrici ritengono che lo Stato abbia assunto il compito far abortire le donne con una finalità assimilabile alla pulizia etnica. Si può avanzare l'ipotesi che lo Stato abbia compiuto anche un ragionamento pratico: facendo sparire la prova della violazione e salvando quindi le apparenze, le donne sarebbero state più facilmente accettate dalle famiglie. Recentemente, si è cominciato a riflettere sulle gravidanze forzate come ad un grave crimine contro l'infanzia mettendo al centro le sofferenze di questi bambini che, quando riescono a sopravvivere, sono spesso condannati a privazioni di ogni genere e a un'esistenza ai margini della società. CARPENTER, Robyn C., «Surfacing Children: Limitations of Genocidal Rape Discourse», in Human Rights Quarterly, 3/2000, pp. 428-477; CARPENTER Robyn C. (ed.), Born of War: Protecting Children of Sexual Violence Survivors in Conflict Zones, Bloomfield, Kumarian Press, 2007; GOODHART, Michael, «Sins of the Fathers: War Rape, Wrongful Procreation, and Children's Human Rights», in Journal of Human Rights, 3/2007, pp. 307-324.). Sui significati e sui meccanismi di rigetto, rimozione e occultamento di questi "figli delle vergogna" al di fuori del subcontinente indiano cfr. FALCOMER, Andrea, "Gli "orfani dei vivi". Madri e figli della guerra e della violenza nell'attività dell'Istituto San Filippo Neri (1918-1947)»in Dep 10, maggio 2009, pp. 76-93; di LENTIN, Ronit, «Lo stupro della nazione: le donne "raccontano" il genocidio» in Dep, 10, maggio 2009, pp. 153-168. 
sono sottoposte ad un "controllo medico completo", sinonimo di aborto, che all'epoca in India è illegale ${ }^{37}$. Se invece le gravidanze sono a uno stadio avanzato si tengono appartate le future madri fino al momento dal parto. Esse lasceranno poi i neonati nell'ashram. Solo dopo aver abbandonato i loro figli queste donne potranno essere presentate alle famiglie ${ }^{38}$.

Che ne è dei bambini nati prima che le donne siano rintracciate39? Le donne ritrovate in Pakistan subiscono forti pressioni affinché lascino indietro i figli. Talvolta li portano nel campo di Jalandhar e lì sono concessi loro quindici giorni di tempo per decidere se separarsi o meno dai figli40. Da questa decisione dipende la loro opportunità di ritorno in famiglia.

La lacerazione provocata dall'abbandono di questi bambini è profonda sia per le madri, sia per le operatrici che, se da un lato contribuiscono a portare avanti i piani del governo, dall'altro sono fortemente scosse dalla sofferenza che queste scelte (obbligate) procurano. Anche a causa della presenza di questi bambini, man mano che il tempo passa, i recuperi divengono sempre più difficili e nel 1954 si decide di porre termine ai rimpatri forzati ${ }^{41}$.

Molti problemi derivano dalla definizione di abducted person fissata per legge (Abducted Persons Recovery and Restoration Ordinance divenuta legge nel 1949). In base alla norma si considerano abducted persons i maschi fino ai 16 anni di età e le femmine di qualsiasi età, di religione musulmana [ci si riferisce alla legge relativa ai compiti di recupero in territorio indiano, nda], che tra il $1^{\circ}$ marzo 1947 e il $1^{\circ}$ gennaio 1949 erano stati separati dalla famiglia e si sono trovati a vivere con o sotto il controllo di qualsiasi altro individuo o famiglia. La legge include i bambini nati dopo il $1^{\circ}$ marzo $1947^{42}$. La rigida e meccanica definizione di abducted person non tiene conto di una

37 MENON, Ritu, BHASIN, Kamla, « Abducted Women and the Question of Honour », cit., pp. 18-19.

${ }^{38}$ BUTALIA, Urvashi, The Other Side of Silence. Voices from the Partition of India, cit., p. 268.

39 Sulla tragedia dei bambini della Partition, assassinati, abortiti, abbandonati dalle madri presso la casa paterna o in orfanotrofi, spesso senza identità, andrebbe approfondita la ricerca e la riflessione. Purtroppo è ancora più difficile che nel caso delle abducted women reperire testimonianze dirette, sia per il silenzio che li ha sempre circondati, sia per l'impossibilità da parte loro di risalire alle origini delle loro storie, sia per il desiderio di celare le loro identità sbagliate. Alcuni importanti riferimenti sono ad ogni modo presenti in BUTALIA, Urvashi, The Other Side of Silence. Voices from the Partition of India, cit., che dedica loro un capitolo, e MENON, Ritu, BHASIN, Kamla, Borders \& Boundaries. Women in India's Partition, cit., passim.

40 MENON, Ritu, BHASIN, Kamla, Borders \& Boundaries. Women in India's Partition, cit., pp. 100-101.

${ }^{41}$ BUTALIA, Urvashi, The Other Side of Silence. Voices from the Partition of India, cit., p. 164.

42 Abducted Persons (Recovery and Restoration ) Act, 1949. Il testo di questa legge si può reperire in MENON, Ritu, BHASIN Kamla, Borders \& Boundaries. Women in India's Partition, cit., pp.261-263. 
quantità di situazioni particolari: chi per esempio, dopo tale data si converte o si sposa per scelta con un membro dell'altra comunità, chi è rapita e stuprata da un correligionario, chi, per svariati motivi, ritenga meglio per sé accettare la situazione. La prima risoluzione dell'Indian National Congress, prevede che alle persone recuperate sia data "ogni opportunità di ritornare alla vita di sua scelta" 43 , ma tale opportunità scompare completamente dalle successive azioni dei governi e dalla norma. Per legge, invece, è prescritto l'automatico annullamento del matrimonio, anche se la donna rapita non lo desidera; per legge una donna è sempre considerata "sequestrata" e il suo rimpatrio, una volta ritrovata, obbligatorio. I figli nati da un matrimonio considerato nullo sono illegittimi. Ma soprattutto per legge la persona ritenuta "abducted" da un ufficiale di polizia, deve essere presa in custodia e al più presto trasferita in un centro del suo paese di appartenenza religiosa. In breve, dopo aver subito varie forme di violenza, le donne si trovarono intrappolate in un meccanismo normativo che, ideato per aiutarle e dar loro giustizia, finisce per costruire una tipologia di donna permanentemente minore e di conseguenza priva di quei diritti fondamentali che sono costituzionalmente garantiti a tutti i cittadini indiani44. La legge di fatto asseconda la necessità dello Stato di rispondere al proprio punto d'onore riappropriandosi di un proprio bene [le nostre donne] e di liberarsi della presenza contaminante delle donne dell'Altro.

Su entrambi i fronti ci sono donne che manifestano apertamente la loro contrarietà ad essere rimpatriate, ma la loro restituzione diventa una questione di prestigio per le due nazioni e oscura gli aspetti umanitari dell'impresa45.

Se da un lato c'è accordo e collaborazione tra India e Pakistan sul recupero e rimpatrio delle donne, dall'altro esso diviene parte della competizione tra i due Stati, non solo nel voler dimostrare una maggiore civiltà, attraverso lo zelo delle operazioni di recupero, ma anche, al contrario, usando le donne come ostaggi. Illuminante, a questo riguardo è la vicenda di una donna musulmana volontariamente fuggita con un indù che ha in seguito sposato. Il Pakistan fa del suo recupero una questione nazionale e afferma che non contribuirà più a restituire le donne indù se la loro ragazza non viene

\footnotetext{
43 Vedi nota 8.

44 MENON, Ritu, BHASIN Kamla, Borders \& Boundaries. Women in India's Partition, cit., p. 106. La legge è approvata nel dicembre del 1949 e la Costituzione indiana entra in vigore il mese successivo.

45 MENON, Ritu, BHASIN, Kamla, «Abducted Women and the Questions of Honour», cit., p. 17. Si veda anche la dichiarazione di Mridula Sarabhai, la responsabile del progetto di recupero, citata a pag. 22: «recovery work is not only a humanitarian problem, it is a part of my political ideology».
} 
tornata indietro. Le pressioni sono tali che la giovane alla fine cede alla ragion di Stato ${ }^{46}$.

India e Pakistan danno per scontato che una donna sequestrata durante la Partition non possa desiderare altro che essere allontanata dai suoi violentatori ed essere riportata a casa47; ciò per moltissime donne è vero, e diviene parte della costruzione ideologica sottesa alla Recovery Operation che si scontra tuttavia con altri dati di realtà di cui non si vuole tenere conto. Tra i politici si alza qualche voce discordante, come quella di Shri Mahavir Tyagi per il quale tali recuperi sono dei veri e propri sequestri di persona ${ }^{48}$, e di chi mette in evidenza che a queste donne vengono negati dei diritti fondamentali come quello di comparire davanti a un giudice (habeas corpus), o di scegliere se vogliono vivere in India e non essere rinchiuse in un campo e deportate in Pakistan ${ }^{49}$ in base alla semplice decisione di un poliziotto, ma cadono nel vuoto ${ }^{50}$.

È così che le istituzioni si trovano di fronte a donne che non intendono essere recuperate e riportate a casa. Quale casa poi? Le famiglie si trasferiscono in quella che è designata come la loro patria avendo come punto di riferimento la religione, ma in questo modo milioni di persone vengono in realtà allontanate da casa sicché, paradossalmente, le donne rapite rimangono più vicine a casa rispetto ai loro familiari profughi in campi distanti centinaia di chilometri.

Differenti sono le esperienze di sequestro e violenza che le donne subiscono e differenti sono le ragioni per cui parte delle donne rifiutano, o tentano di rifiutare il recupero forzato. I governi non considerano, per esempio, una serie di casi fortunati, donne che si sono trovate bene con i loro rapitori o, quantomeno, con i quali hanno costruito una qualche forma di rapporto e una famiglia51. Urvashi Butalia fa notare che quello è un contesto socio-culturale in cui i matrimoni vengono combinati dalle famiglie, tutte le famiglie: indù, sikh e musulmane, e dove non si tiene conto dei desideri degli sposi. La maggior parte dei matrimoni [che, fra l'altro, implicano tradizionalmente per la ragazza il distacco definitivo dalla famiglia di origine, nda] può essere considerato come un rapimento, un assalto da parte di uomini ${ }^{52}$, parte di quel continuum di violenza cui si è accennato all'inizio. La differenza risiede nel fatto che lo

\footnotetext{
46 DAS, Veena, op. cit., pp. 80-81.

47 MENON, Ritu, BHASIN, Kamla, Abducted Women and the Questions of Honour, cit., p. 23.

48 Ibidem, p. 24.

49 Si tratta del dibattito intorno al recupero e l'invio in Pakistan delle donne musulmane, le indù e sikh al di là del confine vivono la medesima condizione di persone senza diritti.

5o MENON, Ritu, BHASIN, Kamla, Borders \& Boundaries. Women in India's Partition, cit., p. 106.

${ }^{51}$ BUTALIA, Urvashi, The Other Side of Silence. Voices from the Partition of India, cit., pp. 147148.

${ }^{2}$ Ibidem.
} 
sposo ha una religione diversa, ma non è detto che ciò sia decisivo in un contesto di pratiche devozionali spesso condivise. Il distacco dalla famiglia d'origine ha sì un carattere di estrema violenza in occasione della Partition, ma se lo si osserva dal punto di vista dello spazio di manovra e di scelta concessi alle spose, esso può non allontanarsi molto della normale esperienza di una donna in tempi di pace.

Non riuscendo a distinguere tra abusanti e "salvatori" alcune donne si dimostrano molto diffidenti nei confronti di chi vuole portarle via, temendo di essere nuovamente vendute53.

Utilizzando l'argomentazione dell'onore una ragazza giovane e brillante portata nel campo di Kujra, dove venivano raccolte le donne ritrovate nell'area di Sialkot, Gujrat e Jhelum, ribalta asserzione di Mridula Sarabhai circa l'immoralità di quei rapimenti, dicendole che se è vero che il rapimento è immorale e che lei è venuta a salvarle, è oramai troppo tardi, perché ci si sposa una sola volta. Vuole forse che si risposino? Questo non è forse immorale? La ragazza mette in risalto un altro aspetto importante: l’incapacità dei loro parenti di proteggerle dal rapimento54. La conservazione dello status di donna sposata, praticamente l'unico socialmente ammissibile per una donna, di fronte alle prospettiva di vivere in condizioni meno onorevoli, ma altresì il rancore verso chi non ha saputo o voluto salvarle giocano un ruolo importante nel motivare il rifiuto di rientrare.

Un attacco diretto all'autorità dello Stato proviene da un gruppo di ragazze convertite all'Islam, per le quali esso ha perso ogni diritto d'intervenire nelle loro vite dato che non è stato in grado di prevenire le brutalità della Partition. Incapace di difenderle, come può ora lo Stato costringerle a far qualcosa55?

Anche una volta rimpatriate, alcune donne manifestano la loro resistenza con scioperi della fame o il rifiuto di cambiare il modo di vestirsi, ma a poco valgono le proteste contro il meccanismo che lo Stato ha messo in moto ${ }^{56}$.

\section{Abducted women contro donne morte con onore}

\footnotetext{
53 Ibidem, p. 150.
}

54 Questa ragazza, dopo aver opposto un'ostinata resistenza al rimpatrio, viene fatta incontrare con la madre, portata appositamente dall'India e alla fine deciderà di riunirsi alla famiglia. BASU, Aparna, op. cit., p. 131.

55 Ibidem.

${ }^{56}$ MENON, Ritu, BHASIN, Kamla, Abducted Women and the Questions of Honour, cit., p. 15. 
La posizione delle donne rapite all'interno del sistema dell'onore può essere compresa meglio se la si raffronta con quella delle donne morte con onore e alla considerazione riservata a queste ultime.

Nonostante il pericolo sia incarnato dall'Altro, le donne non devono guardarsi solo da esso, ma anche dalla violenza dei loro parenti stretti. Accade infatti che in comunità accerchiate dai musulmani - le informazioni si riferiscono prevalentemente ai sikh gli uomini decidano di uccidere le loro figlie, madri, sorelle, cugine, mogli anziché vederle cadere nelle mani dei nemici. Gurmeet Singh narra di come il loro villaggio, Thamali57, sia stato circondato, e gli abitanti abbiano deciso di riunire le donne e i bambini nel gurudwara, uccidere tutti e dare poi fuoco al tempio ${ }^{58}$.

Urvashi Butalia riporta le memorie di Mangal Singh, settantenne alla fine degli anni Ottanta, che insieme con i fratelli mette a morte le donne e i bambini della propria famiglia, diciassette persone. Nel cercare di spiegarne le motivazioni egli evita di utilizzare termini come assassinati preferendo ad essi la parola martirizzati. Insiste sul fatto che le donne "si erano offerte" perché la morte è preferibile a ciò che sarebbe altrimenti accaduto: la conversione e lo stupro. Incalzato da chi gli chiede come sia stato possibile, egli risponde rabbiosamente "You know this race of Sikhs?" Le donne, racconta sono scese nel cortile e hanno chiesto di essere martirizzate insieme ai loro piccoli:

The real fear was one of dishonour, if they have been caught by the Muslims, our honour, their honour would have been sacrificed, lost. It's a question of one's honour... if you have pride you do not fear59.

La paura è non già quella di vedere morire tra terribili sofferenze i propri cari ma anzi, che sopravvivano, e attraverso la violazione dei loro corpi, l'onore maschile di tutta la comunità venga macchiato. Anche la conversione delle donne e dei bambini ad altre religioni diventa una ragione di disonore che giustifica il ricorso all'omicidio. Assassinii che vengono prospettati come atti di pietà, sollecitati dalla richiesta dalle stesse vittime come, nel caso di Virsa Singh, che spara a 50 donne: alla propria moglie e ad altre donne del vicinato che gli si fanno intorno dicendo "Viran, pehle mannu maar"

57 Villaggio nel distretto di Rawalpindi, Pakistan.

${ }^{8}$ BUTALIA, Urvashi, The Other Side of Silence. Voices from the Partition of India, cit. p. 205.

59 Ibidem, pp. 194-195. 
(Fratello uccidi me per prima). Virsa Singh diventerà in seguito un medico e si rifarà una famiglia, nessun rimorso nel suo ricordo ${ }^{60}$.

A volte, chi effettivamente agisce o contribuisce alla morte delle donne di famiglia non ammette il proprio ruolo: Iqbal racconta di essere stato testimone dell'omicidio, ad opera del marito, della cugina avvenuto per strangolamento con la dupatta [la sciarpa, parte essenziale dell'abbigliamento femminile] ma non dice che ne aveva stretto un capo e che quindi aveva contribuito attivamente all'uccisione della giovane donna. Anche in questo frangente si riporta che la decisione di morire sia stata della vittima, rafforzata dall'argomento che se gli uomini fossero stati uccisi nessuno avrebbe protetto le donne «They had no choice» ${ }^{61}$.

Oltre alle donne che richiedono per sé e per i figli di essere uccise vi sono quelle che si uccidono da sé ingerendo l'afim [oppio] ${ }^{62}$. Alcune, con la prospettiva che la morte possa giungere in ogni momento, portano dei pacchetti di veleno legati al collo ${ }^{63}$. Non risulta che un tale equipaggiamento sia previsto per gli uomini. Un ospedale rifornisce di veleno le donne che ne fanno richiesta; altre scelgono di lanciarsi dai ponti64.

Uno degli episodi più celebrati è quello del suicidio di massa delle donne di Thoa Khalsa ${ }^{65}$ che, insieme ai loro figli, si gettano nel pozzo del villaggio. Il pozzo si riempie a tal punto che le ultime non riescono a portare a termine il progetto di morte ${ }^{66}$. Questo episodio diventa un topos della narrazione sul coraggio e il senso dell'onore dei sikh ${ }^{67}$.

Alla fine degli anni Ottanta, Urvashi Butalia è ancora testimone del rispetto guadagnato dalla comunità di sopravvissuti di Thoa Khalsa (ora dislocati a Jangpura e Bhogal, a Delhi) in conseguenza dell'eroica morte delle loro donne ${ }^{68}$. In queste narrazioni non manca il richiamo mitico al jahuar: costume in base al quale le donne

${ }^{60}$ MENON, Ritu, BHASIN, Kamla, Borders \& Boundaries. Women in India's Partition. cit., pp. 49-50.

61 Ibidem, p. 51.

62 BUTALIA, Urvashi, The Other Side of Silence. Voices from the Partition of India, cit. p. 198.

${ }^{63}$ MENON, Ritu, BHASIN, Kamla, Borders \& Boundaries. Women in India's Partition, cit., p. 46.

64 Ibidem, p. 51.

65 Distretto di Rawalpindi, Pakistan.

${ }^{66} \mathrm{Si}$ veda il racconto della sopravvissuta Basant Kaur in BUTALIA, Urvashi, The Other Side of Silence. Voices from the Partition of India, cit. p. 198-202.

${ }_{67} \mathrm{Se}$ si visita il sito web SikhiWiki, enciclopedia della cultura sikh, vi si ritroverà la testimonianza di una delle superstiti del suicidio di massa, così come riportata dalla ricerca di Urvashi Butalia, da cui è stato rimosso però il commento critico dell'autrice.

URL: < http://www.sikhiwiki.org/index.php/1947_Massacre_at_Thoa_Khalsa >[consultato il 16 agosto 2011].

${ }^{68}$ BUTALIA, Urvashi, The Other Side of Silence. Voices from the Partition of India, cit. p. 197-8. 
rajput, di fronte alla sconfitta del regno, si danno alla morte collettiva immolandosi su una pira ${ }^{69}$.

La santificazione delle donne che si sono sacrificate per salvare l'onore delle comunità non risparmia neanche il Mahatma Gandhi il quale, pur difendendo la causa dell'accoglienza delle abducted women, si esprime con i seguenti toni riguardo alle donne suicide:

I have heard that many women who did not want to lose their honour chose to die. Many men killed their own wives. I think that is really great, because I know that such things make India brave. After all, life and death is a transitory game. Whoever might have died are dead and gone; but at least they have gone with courage. They have not sold away their honour. Not that their lives were not dear to them, but they felt it was better to die with courage rather than be forcibly converted to Islam by the Muslims and allow them to assault their bodies. And so those women died. They were not just a handful, but quite a few. When I hear all these things, I dance with joy that there are such brave women in India ${ }^{70}$.

Colpisce la sua adesione all'idea che il mantenimento della purità e della religione da parte delle donne richieda il sacrificio della vita. In questo contesto va, a onore del vero, inserita la profonda riflessione che Gandhi sta svolgendo sulla nonviolenza del forte, quella di chi, scegliendo di non usare le armi, si oppone con coraggio all'assaltatore, accettando di morire senza uccidere ${ }^{71}$. In questa situazione, tuttavia, il Mahatma sembra allinearsi al senso comune. Egli loda non tanto la nonviolenza delle donne - e chi si sarebbe aspettato o avrebbe addirittura auspicato una reazione violenta da loro? - ma la loro attiva reazione di fronte alla prospettiva di essere disonorate ovvero, di divenire il veicolo del disonore dei loro uomini - e convertite forzatamente. Mentre da parte degli uomini sikh accettare una morte non-violenta, anche suicida, senza usare violenza contro il nemico, sarebbe un'azione in linea con il tipo di rivoluzione che il Mahatma propone, la morte coraggiosa delle donne per non divenire il bottino di guerra di altri uomini è quanto ci si aspetta da esse in base al tradizionale

69 DAS,Veena, op. cit., p. 63. A sua volta il suicidio di massa delle donne rajput si può ricollegare al costume del sati, l'immolazione delle vedove sulla pira funeraria del marito.

${ }^{70}$ GANDHI, Mohandas K., « Speech at Prayer Meeting », in cit., vol. 96., pp. 388-389.

${ }^{71}$ Gandhi trae spunto per le sue argomentazioni sulla non-violenza del forte facendo riferimento alla lotta dei Pathan guidati da Khan Abdul Ghaffar Khan che negli anni '3o conducono una lotta disarmata contro il regime coloniale britannico a costo di gravi perdite. I Pathan, pur provenendo da una tradizione marziale, scelgono di rinunciare alle armi e di fare della loro disponibilità a morire con coraggio un punto centrale della rielaborazione del loro concetto di onore. 
codice d'onore pronto a sacrificarle per tenere saldi i principi patriarcali. Butalia fa notare come le donne che chiedono di farsi uccidere o si suicidano diventino il simbolo del coraggio e della mascolinità sikh, quando sono però loro, e non gli uomini, a morire ${ }^{72}$.

$\mathrm{Su}$ questo affresco che tramanda una memoria di valore, onore e martirio, cominciano tuttavia ad aprirsi alcune crepe, allargate dalla ricerca delle storiche. Forti dubbi vengono avanzati sull'assoluta volontarietà delle donne a sacrificarsi. La giustificazione proposta da Iqbal circa la spontanea ricerca della morte da parte delle donne e l'assenza di una pressione maschile nella vicenda, viene messa in discussione dalla stessa moglie che durante l'intervista lo interrompe dicendo "Devono averle incoraggiate, dopo tutto, cosa possono fare delle donne in questa situazione? Devono averle persuase..."73 e con l'approfondirsi delle interviste emergono storie di donne che si rifiutano di prendere il veleno consigliato loro: «We tried to persuade Veeran, a young cousin to take opium, but she refused»74. Le donne che rifiutano di uccidere se stesse e le proprie figlie sono poi disprezzate e considerate codarde 75 . Ciò dà un'ulteriore conferma di quanto l'atto di porre fine alla propria vita sia considerato dovuto, più che volontario, e aggiunge perplessità sulla libera scelta di queste donne.

Se si esaminano le vicende di omicidio-suicidio, si nota che in molti casi le donne sono uccise per la deliberata scelta dei soli uomini anche se si tramanda che esse lo abbiano chiesto e, a parte la censura su chi rigetta l'opzione della morte, non si sa quante siano state effettivamente forzate a togliersi la vita. Per forzate non s'intende solo chi è costretta fisicamente ma chi, in un contesto in cui la vita di una donna senza uomini è impensabile, sia psicologicamente, sia materialmente, dove si è educate a pensare che la propria esistenza sia lecita solo ricoprendo il ruolo di moglie-madre all'interno della propria comunità, non è difficile capire che per una donna in quella situazione la morte sia l'unica opzione concepibile.

Pur nell'ambito costrittivo del sistema dell'onore, lo spazio di manovra e negoziazione è comunque sbilanciato a favore degli uomini, come dimostrano le vicende della famiglia di Charanjit Singh Bhatia: lo zio di questi, padre di sei ragazze, vive nell'area di Quetta, nell'attuale Pakistan nord-occidentale, in ottimi rapporti con i suoi vicini musulmani. $\mathrm{Al}$ momento dello scoppio delle violenze questi gli propongono di combinare dei matrimoni tra i loro figli e le sue ragazze. In tal modo tutta la famiglia

\footnotetext{
${ }^{72}$ BUTALIA, Urvashi, The Other Side of Silence. Voices from the Partition of India, cit. p. 209. 73 MENON, Ritu, BHASIN, Kamla, Borders \& Boundaries. Women in India's Partition, cit., p. 51.

74 Ibidem, p. 51.

75 Ibidem, p. 54.
} 
sarà protetta. Lo zio sembra accettare la proposta, ma quella sera stessa riunisce tutta la sua famiglia, tredici persone, sul tetto della casa, le uccide, dà loro fuoco e si suicida. Il capofamiglia preferisce una morte reale a quella simbolica che tali matrimoni significherebbero per lui. In un altro ramo di quella stessa famiglia invece, si decide di lasciare indietro una figlia in cambio di un passaggio sicuro per l'India, mentre altre tre ragazze vengono rapite nella confusione del momento e in seguito recuperate e reintegrate ${ }^{6}$.

${ }^{76}$ Ibidem, p. 48. 


\section{Chi è degna di essere ricordata?}

Nel corso di un'intervista, Reva, ricordando la cugina Krishna assassinata dai suoi parenti perché non cadesse nelle mani dei musulmani, afferma che le donne morte in queste circostanze sono ricordate con orgoglio dai familiari - anche dalle donne - che ne narrano le storie e ne mostrano le foto ${ }^{77}$.

Mentre nelle narrazioni familiari le memorie sulle donne uccise, possibilmente suicide, proliferano, il silenzio cala quelle rapite o che si sono rifiutate di morire con onore. Veena Das mette in evidenza come una censura collettiva sia scesa sulle donne che sono rimaste in Pakistan e hanno accettato un matrimonio con un musulmano ${ }^{78}$. I sopravvissuti di Thoa Khalsa giungono a negare che le donne del loro villaggio siano presenti nell'elenco di quelle sequestrate, giacché risultano tutte suicide, ma ciò viene confutato dai fatti: nomi di donne rapite compaiono lì come in tanti villaggi; d'altra parte come potrebbero i superstiti giustificare il martirio di tante donne, se si venisse a sapere che altre sono state comunque violate 79 ?

Partendo proprio dal silenzio di una famiglia circa la sparizione di due sorelle, da tutti risaputa, ma che i familiari si vergognano di menzionare, Urvashi Butalia sviluppa la sua ricerca sulle storie che stanno dietro questi silenzi, «the stories that describe the dark side of the freedom» 80 .

Si è dunque stabilito uno spartiacque su chi merita di essere ricordato e chi no, una memoria che seleziona sulla base dell'aderenza femminile alle norme sull'onore.

Il racconto di Jyotirmoyee Devi, Shei Chheleta (Quel ragazzino) ${ }^{81}$, illustra con esemplare chiarezza come l'idea della conservazione della purità sessuale sia il perno di tutto il discorso - e dei silenzi - sulla memoria delle violenze di genere durante la Partition e vale la pena soffermarcisi un momento. Nel corso degli scontri avvenuti a Lahore, la piccola Raj e la sua famiglia si mettono in salvo, ma la madre rimane indietro. Una volta in salvo, partono le ricerche della donna da cui risulta che è stata uccisa. Malgrado i rapporti sui rapimenti e sugli stupri si dà per certo che sia morta in

\footnotetext{
77 Ibidem, p. 53.

${ }^{78}$ DAS,Veena, op. cit., p. 63.

79 BUTALIA, Urvashi, The Other Side of Silence. Voices from the Partition of India, cit. p. 218219

8oIbidem, p. 134.

${ }^{81}$ Il racconto dell'autrice bengalese Jyotirmoyee Devi (1894-1988) Shei Chhleta viene pubblicato in India per la prima volta nel 1961. Esiste una traduzione in inglese di Debali Mookerjea, «That Little Boy: An English Translation of Jyotirmoyee Devi's Bengali Short Story 'Shei Chhleta'», in Meridians, 2/2002, pp. 128-145.
} 
modo onorevole. Sette anni dopo Raj incontra per caso una mendicante che si rivela essere la madre. La donna è accompagnata da un ragazzino. Nel corso della conversazione Raj comprende che la donna è stata stuprata. La presenza di quel ragazzino, prova vivente della violazione, rende difficile accettare la madre e la scomoda realtà che porta con sé. Raj guadagna tempo e decide che il giorno dopo porrà degli ulteriori quesiti alla mendicante per chiarire i dubbi. Non sarà però più possibile ritrovare la donna e il ragazzino. La consapevolezza della violenza subita dalla madre, e l'imbarazzante presenza di quel bambino sbagliato provocano un malessere maggiore della precedente idea che la donna fosse morta ${ }^{82}$.

In conclusione, le vicende che si articolarono intorno al recupero e reintegro delle abducted women mettono in evidenza come lo Stato indiano e la comunità, forzatamente ed erroneamente identificata con la sola componente sikh-indù, si muovono su binari solo apparentemente divergenti. Tenendo l'onore patriarcale come punto di riferimento, il compito dello Stato è quello di recuperare le donne considerate di sua proprietà in base alla loro appartenenza religiosa, mentre le famiglie mantengono il tradizionale controllo sulla sessualità femminile a costo di rigettare quelle donne che non rispondono o si sospetta che non rispondano più a questo canone. Laddove è possibile, lo Stato agisce rimuovendo i segni della contaminazione per mantenere l'apparenza della purità delle donne recuperate, altrimenti assorbe le donne e i figli inaccettabili nelle proprie istituzioni. Il reintegro delle abducted women avviene a prezzo della censura su ciò che hanno passato, assecondata dall'adesione delle stesse vittime alle norme dell'onore patriarcale. Di fronte a questo silenzio stride la memoria delle donne martirizzate per salvare l'onore della comunità. Si tratta di una memoria amplificata e reiterata nelle commemorazioni, adulterata nell'evidenziare il senso di sacrificio delle donne senza menzionare le pressioni e i presupposti culturali che hanno portato a questi atti. È altresì una memoria selettiva che occulta i casi di resistenza e i deliberati omicidi di familiari innocenti da parte dei loro uomini.

Il ricordo delle abducted women viene conservato dalla memorialistica e dalla narrativa fino a quando, alla fine degli anni Ottanta, nuovi filoni di ricerca slegati dalla retorica nazionalista ufficiale, non fanno affiorare le loro storie. Ciò non rende solo loro giustizia ma, partendo da un approccio storiografico apparentemente di nicchia come quello femminista, permette di rimettere in discussione nei suoi temi e assunti generali tutta la storiografia della Partition.

82 MOOKERJEA-LEONARD, Debali, «Quarantined: Women and the Partition», cit., p. 37. 


\section{* L'autore}

Piera Cavenaghi è dottoranda di ricerca (PhD Student) in Storia, culture e strutture delle aree di frontiera presso il Dipartimento di Studi Umani dell'Università di Udine; sta sviluppando un progetto di ricerca sul tema degli honour killings nelle comunità immigrate in Italia. Nel 2007 ha conseguito un dottorato di ricerca presso l'Università la Sapienza di Roma con una tesi dal titolo Origine e sviluppo del nazionalismo pukhtun tra Afghanistan e India dal periodo coloniale alla metà del Novecento.

URL: < http://studistorici.com/progett/autori/\#Cavenaghi >

\section{Per citare questo articolo:}

CAVENAGHI, Piera, "Lasciar parlare il silenzio. La Partition del subcontinente indiano e le abducted women», Diacronie. Studi di Storia Contemporanea: Le sembianze di Clio: approcci alla storia, 29/10/2011, URL:< http://www.studistorici.com/2011/10/29/cavenaghi_numero_8/>

\section{Diacronie Studi di Storia Contemporanea $\beta$ www.diacronie.it}

Risorsa digitale indipendente a carattere storiografico. Uscita trimestrale. redazione.diacronie@hotmail.it

Comitato di redazione: Marco Abram - Giampaolo Amodei - Jacopo Bassi - Luca Bufarale - Alessandro Cattunar - Alice De Rensis Barbara Galimberti - Deborah Paci - Fausto Pietrancosta - Martina Sanna - Matteo Tomasoni - Luca Zuccolo 